\title{
ANÁLISE ETOLÓGICA RELACIONADA AO PROCEDIMENTO DE DESGASTAR OU NÃO DENTES DE LACTENTES SUÍNOS
}

\section{ETHOLOGICAL ANALYSIS RELATED TO THE PROCEDURE OF WEARING OR NOT THE TEETH OF PIGLETS IN MATERNITY}

\author{
Gisele Dela Ricci ${ }^{*}$ \\ Osmar Antonio Dalla Costa ${ }^{2}$ \\ Messias Alves da Trindade Neto $^{3}$ \\ Cristiane Gonçalves Titto ${ }^{1}$ \\ ${ }^{1}$ Universidade de São Paulo, Pirassununga, SP, Brasil. \\ ${ }^{2}$ Centro Nacional de Pesquisa em Aves e Suínos - EMBRAPA, Concórdia, SC, Brasil. \\ 3Universidade de São Paulo, São Paulo, SP, Brasil. \\ *Autora para correspondência - giseledelaricci@usp.br
}

\section{Resumo}

O objetivo deste estudo foi avaliar o comportamento de leitões e porcas, frente ao tratamento de desgaste ou não de dentes do lactente. Foram utilizadas 67 matrizes suínas e 827 leitões. O experimento foi realizado em três locais e a obtenção de dados comportamentais foi a partir de etograma, na forma dirigida. As observações dos leitões foram realizadas de dez em dez minutos, durante quatro horas, três vezes por semana. Os dados foram analisados em delineamento inteiramente ao acaso com medidas repetidas no tempo. Utilizou-se o modelo misto com o efeito fixo de tratamento, turno, período e suas interações; e o efeito aleatório de lote utilizando o procedimento MIXED do SAS (2008). Foram testadas 15 estruturas de variância e covariância, escolhendo-se a que apresentou menor valor para o Critério de Informação de Akaike (AIC). Não foram encontrados efeitos significativos para o comportamento agonístico, estereotipado, lúdico e exploratório em relação aos tratamentos assim como para todo o período avaliado $(\mathrm{P}>0,05)$. Efeitos significativos foram identificados no comportamento de mamar, comparando-se os tratamentos $(\mathrm{P} \leq 0,05)$. Não foram encontrados efeitos significativos para o comportamento alimentar das matrizes $(\mathrm{P}>0,05)$. Comer diferiu de beber, para o período avaliado $(\mathrm{P} \leq 0,05)$. Com este estudo, conclui-se que o procedimento de desgaste ou permanência dos dentes de leitões pode influenciar em comportamentos importantes, como o de mamar, responsável pelo eficiente ganho de peso. O desgaste dos dentes pode ser desnecessário diante dos ferimentos causados, em decorrência do manejo errôneo desta pratica. Contudo, há necessidade de mais estudos direcionados a este tema, buscando maiores elucidações.

Palavras-chave: adaptação; bem-estar; comportamento; etologia; lesão. 


\begin{abstract}
The objective of this study was to evaluate the behavior of piglets and sows regarding the treatment of wearing or not the teeth of the piglets. Sixty-seven swine matrices and 827 piglets were used. The experiment was performed in three locations and the behavioral data was obtained from the ethogram, in the directed form. Piglets were observed every ten minutes for four hours, three times a week. The data were analyzed in a completely randomized design with repeated measures in time. The mixed model was used with the fixed treatment effect, shift, period and their interactions, and the random batch effect using the MIXED procedure of SAS (2008). Fifteen structures of variance and covariance were tested, choosing the one that presented the lowest value for the Akaike Information Criterion (AIC). No significant effects were found for the agonistic, stereotyped, playful, and exploratory behavior in relation to the treatments as well as for the whole period evaluated $(\mathrm{P}>0.05)$. Significant effects were identified on the suckling behavior, comparing the treatments $(\mathrm{P} \leq 0.05)$. No significant effects were found for the dietary behavior of the matrices $(\mathrm{P}>0.05)$. The study differed from drinking for the period evaluated $(\mathrm{P} \leq 0.05)$. Permanence or not of the piglets' teeth can affect important behaviors such as suckling, which is responsible for the efficient weight gain. The wear of the teeth may be unnecessary due to the injuries caused as a result of the mismanagement of this practice. More studies on this theme, seeking further elucidations, are necessary
\end{abstract}

Keywords: adaptation; behavior; ethology; injury; welfare.

Recebido em: 28 janeiro de 2016

Aceito em: 31 julho de 2017

\title{
Introdução
}

A prática do corte ou desgaste dos dentes é efetuada até 48 horas de vida dos leitões, buscando reduzir lesões cutâneas nos lactentes e no aparelho mamário da matriz. Apesar dos benefícios da diminuição de lesões cutâneas nos leitões, esse procedimento retira ou fratura o esmalte dentário, causando infecções, decorrentes da exposição da polpa dentária ${ }^{(1)}$. Nos primeiros dias do nascimento, a redução de dentes dos lactentes provoca dor intensa, aguda ou crônica ${ }^{(2)}$.

Em relação às lesões nos tetos das matrizes, o estado de saúde da matriz, especialmente no puerpério, afeta nas disputas pelos tetos e na intensidade das lesões na face dos leitões e no úbere das fêmeas ${ }^{(3)}$. A competição entre os leitões aumenta quando a produção de leite é baixa e existem diferenças de peso entre os lactentes, ou quando o tamanho da leitegada é superior ao número de tetos da fêmea ${ }^{(4)}$.

A maternidade é um ambiente onde se observam comportamentos distintos realizados pelas matrizes e leitões ${ }^{(5)}$. Cada comportamento apresenta sua importância e tem influência direta no bem-estar e desempenho zootécnico. Para promover o conforto e bem-estar das matrizes e leitões, a maternidade deve apresentar prioritariamente grade que evite esmagamentos, fonte de aquecimento com regulagem, piso confortável que evite lesões e capacidade isolante para trocas de calor, temperatura ambiental adequada para leitões e matrizes e limpeza diária ${ }^{(6)}$. 
O suíno possui alta capacidade de aprendizado, curiosidade e amplo aspecto comportamental ${ }^{(7,8)}$. Através da evolução, os suínos aprendem ou herdam adaptações ao ambiente, frustrando-se caso suas expectativas não sejam alcançadas ${ }^{(9)}$. Em ambientes seminaturais, permanecem, diariamente, 52\% do tempo fuçando ou pastando e outros $23 \%$ investigando o ambiente ${ }^{(10)}$. Quando confinados, sem enriquecimentos que permitam exercer comportamentos inerentes à espécie, surge o sentimento de frustração que os direciona ao comportamento investigativo entre si ou na direção dos equipamentos presentes na baia(11).

Porcas lactantes desenvolvem distúrbios comportamentais decorrentes da permanência em locais sem motivação ambiental, mordendo barras da gaiola, demonstrando, por exemplo, desejo de alimentarse. Este distúrbio pode ser maior ou menor, dependendo do tempo de permanência na maternidade, do tipo da instalação utilizada e da ambiência. Do mesmo modo, o confinamento em gaiolas reduz a mobilidade, causando estresse crônico e comprometendo, consequentemente, o bem-estar das matrizes lactantes ${ }^{(12)}$.

Legislações específicas para leitões recém-nascidos já estão descritas e são importantes para a criação adequada dos animais. A legislação da União Europeia (2001) circunscreve a justificativa para a retirada dos dentes do leitão lactente. Segundo a Directiva 91/630/EEC (2001), a recomendação do método e responsabilidade para a adoção se darão quando houver feridas nos tetos das matrizes ou lesões severas de leitões, de modo que tal procedimento assegure a melhoria do bem-estar da matriz e dos lactentes. Outra normativa permite a redução uniforme das pontas dos dentes dos leitões mediante o desgaste ou corte parcial, até o sétimo dia ${ }^{(13)}$.

Diante da importância da etologia e do manejo do desgaste dos dentes na produção de suínos, este estudo teve como objetivo avaliar o comportamento e o desempenho dos leitões e das porcas, frente ao tratamento de desgaste ou não de dentes do lactente.

\section{Material e métodos}

Foram utilizados 67 matrizes suínas e 827 leitões. O experimento foi realizado em três locais: na Embrapa (I), em Concórdia Santa Catarina com clima superúmido e mesotérmico do tipo temperado Cfa com temperaturas médias anuais de $13^{\circ} \mathrm{C}$; o segundo realizou-se na Universidade de São Paulo (II), em Pirassununga, São Paulo, com clima Cwa e temperaturas médias anuais de $23^{\circ} \mathrm{C}$; e na granja comercial Santo Ignácio de Loyola (III), na cidade de Brotas, estado de São Paulo, com clima tropical Cwa e médias anuais de $22{ }^{\circ} \mathrm{C}^{(14)}$.

Os dados foram obtidos na instalação de maternidade, no período compreendido entre o nascimento e o desmame dos leitões, com duração em média de 28 dias, de acordo com o manejo adotado nos locais de experimentais. Entre 10 e 18 horas após o nascimento foram realizados os procedimentos do desgaste dos dentes, a aplicação de ferro dextrano via intramuscular, mossagem, caudoctemia e castração dos leitões machos. A água foi fornecida à vontade para fêmeas e leitões e a ração de acordo com a formulação de cada local experimental para matrizes. 
Os tratamentos direcionados aos leitões lactentes ficaram divididos em: 1) Desgaste do ápice dos dentes incisivos e caninos aos dois dias de idade; 2) Manutenção dos dentes incisivos e caninos intactos. A técnica utilizada para o desgaste foi a que garante maior exposição dos dentes ${ }^{(15,16)}$. $\mathrm{O}$ desgastador utilizado foi Dremel ${ }^{\circledR}$ (EUA), com diferentes níveis de rotação. Após o procedimento de desgaste dos dentes, a cavidade bucal foi avaliada em relação à presença de lesões na gengiva, língua ou dentes ${ }^{(17-19)}$.

Para obtenção de dados comportamentais, foi construído um etograma ${ }^{(20)}$ para amostragem da frequência de realização de cada comportamento dos leitões e da matriz. Para a avaliação do comportamento foram utilizadas as seguintes variáveis: ócio, mamar, comer, beber, estereotipias, agressividade, ludicidade, exploratório. A observação dos leitões e matrizes suínas se deu na forma dirigida $^{(21)}$, de modo a não afetar o comportamento natural dos mesmos. As observações dos leitões foram realizadas de dez em dez minutos, durante quatro horas, três vezes por semana e durante o período de desmame. Na identificação do comportamento, adotou-se etograma elaborado com base no comportamento das matrizes e dos leitões na fase de maternidade. Os observadores foram treinados para identificar os principais pontos comportamentais dos animais, baseando-se em escala previamente definida.

Os dados foram analisados em delineamento inteiramente ao acaso com medidas repetidas no tempo. Utilizou-se o modelo misto com o efeito fixo de tratamento, turno, período e suas interações, e o efeito aleatório de lote utilizando-se o procedimento MIXED do SAS (2008) ${ }^{(22)}$. Foram testadas 15 estruturas de variância e covariância, escolhendo-se a que apresentou menor valor para o Critério de Informação de Akaike (AIC).

Para realização da análise de comportamento das matrizes, foi calculado o percentual de avaliações de cada comportamento durante todo o período de avaliação (de 1 a 4 semanas de idade dos leitões) e aplicada à análise da variância para o modelo contendo os efeitos de granja e tratamento. A comparação de médias ocorreu através da diferença mínima significativa de Fisher (opção DIFF do comando LSMEANS).

\section{Resultados e discussões}

Após o nascimento, os leitões são direcionados a mamar o colostro, rico em imunoglobulinas e importante para sua imunidade passiva e desenvolvimento intestinal ${ }^{(23)}$. Durante este período, os leitões são expostos ao aparelho mamário da porca e, nas primeiras dezoito horas, elegem um teto preferencial, sendo que neste período ocorrem disputas entre indivíduos e cada lactente poderá mamar em três ou quatro tetos diferentes ${ }^{(24,25)}$. Essas disputas são, normalmente, agressivas, causam lesões faciais graves entre os animais com dentes íntegros e submetidos ao desgaste. Portanto, ambas as opções reduzem o bem-estar dos animais nesta fase. Uma vez estabelecida à ordem da mamada, dificilmente ocorrem novas disputas e o leitão ocupará o mesmo teto ou par de tetos até o desmame ${ }^{(26)}$. No entanto, neste estudo, comprovou-se que a permanência dos dentes não aumentou a agressividade entre os lactentes, uma vez que o comportamento agonístico não diferiu estatisticamente $(\mathrm{P}>0,05)$ 
entre os tratamentos. As médias percentuais observadas durante todo o período avaliado caracterizaram maiores frequências para o tratamento de desgaste de dentes (2,95\%) em relação ao tratamento controle $(2,43 \%)$.

A permanência ou retirada dos dentes não teve influência no comportamento estereotipado, uma vez que não houve diferença estatística $(\mathrm{P}>0,05)$. $\mathrm{O}$ comportamento estereotipado está relacionado à monotonia do confinamento verificada nos locais onde o experimento foi realizado, devido à ausência de enriquecimentos ambientais e à restrição do espaço, caracterizando as deficiências ambientais e de manejo que determinam as alterações no bem-estar dos suínos ${ }^{(27)}$. Em ambos os tratamentos foi evidenciado este tipo de comportamento, indicando que as estereotipias estão presentes dentro dos confinamentos e são atividades neutralizadoras adotadas pelos animais, independentemente do tratamento ${ }^{(28)}$.

Não se identificaram diferenças significativas no comportamento exploratório do leitão em função dos tratamentos $(\mathrm{P}>0,05)$. O comportamento exploratório é típico da espécie suína ${ }^{(29)}$, uma vez que o animal possui alta curiosidade pelo ambiente, fuçando ou investigando a baia, os objetos e até mesmo os outros indivíduos.

Efeitos significativos foram identificados no comportamento de mamar, comparando-se os tratamentos empregados $(\mathrm{P} \leq 0,05)$. Os valores médios percentuais observados durante todo o período foram $24,18 \%$ para o tratamento de manutenção dos dentes e 23,97\% para o tratamento de desgaste de dentes. Leitões do tratamento de manutenção dos dentes apresentaram maior peso na entrada e na saída da maternidade, caracterizando-se como mais vigorosos e, frequentemente, com maiores oportunidades de vencer a disputa pelos tetos anteriores, enquanto os mais leves ou subordinados se limitam aos posteriores, normalmente, com menor quantidade de leite ${ }^{(24,30,31)}$.

O comportamento lúdico entre leitões e com a mãe não foi influenciado pelos tratamentos empregados $(\mathrm{P}>0,05)$.

O estudo comportamental demonstrou que, em boa parte do tempo, os leitões apresentaram comportamentos definidos como de ócio (dormir, deitado, em pé e sentado) em ambos os tratamentos avaliados; no entanto, sem ser caracterizada diferença significativa. Avaliando-se o comportamento e parâmetros fisiológicos de leitões nas primeiras 24 horas de vida, Ferreira et al. ${ }^{(32)}$ constataram que, durante 53,8\% do tempo do primeiro dia de vida, os animais dormiam e durante 31,2\% mamavam.

Os lactentes que foram submetidos ao tratamento de desgaste dos dentes exibiram frequentes lesões na boca, oriundas do manejo incorreto, com desenvolvimento de processos inflamatórios, provavelmente indutores de dor aguda e crônica, tornando os animais menos ativos dentro da baia (Figura 1). Os animais do tratamento de manutenção dos dentes apresentaram as mesmas características e consequências; no entanto, os ferimentos oriundos das disputas pelos tetos, durante as primeiras duas semanas, foram mais severos e intensos que no tratamento de desgaste de dentes (Figura 2). Salienta-se que o sistema de confinamento e o espaço restrito das gaiolas permitiam apenas movimentos limitados dos animais. Nesse caso, a ausência de enriquecimento ambiental, tornou o ambiente monótono para os animais de ambos os tratamentos, caracterizando restrição para o bemestar dos suínos. O período em que os animais foram avaliados não diferiu estatisticamente em relação aos tratamentos $(\mathrm{P}>0,05)$. 

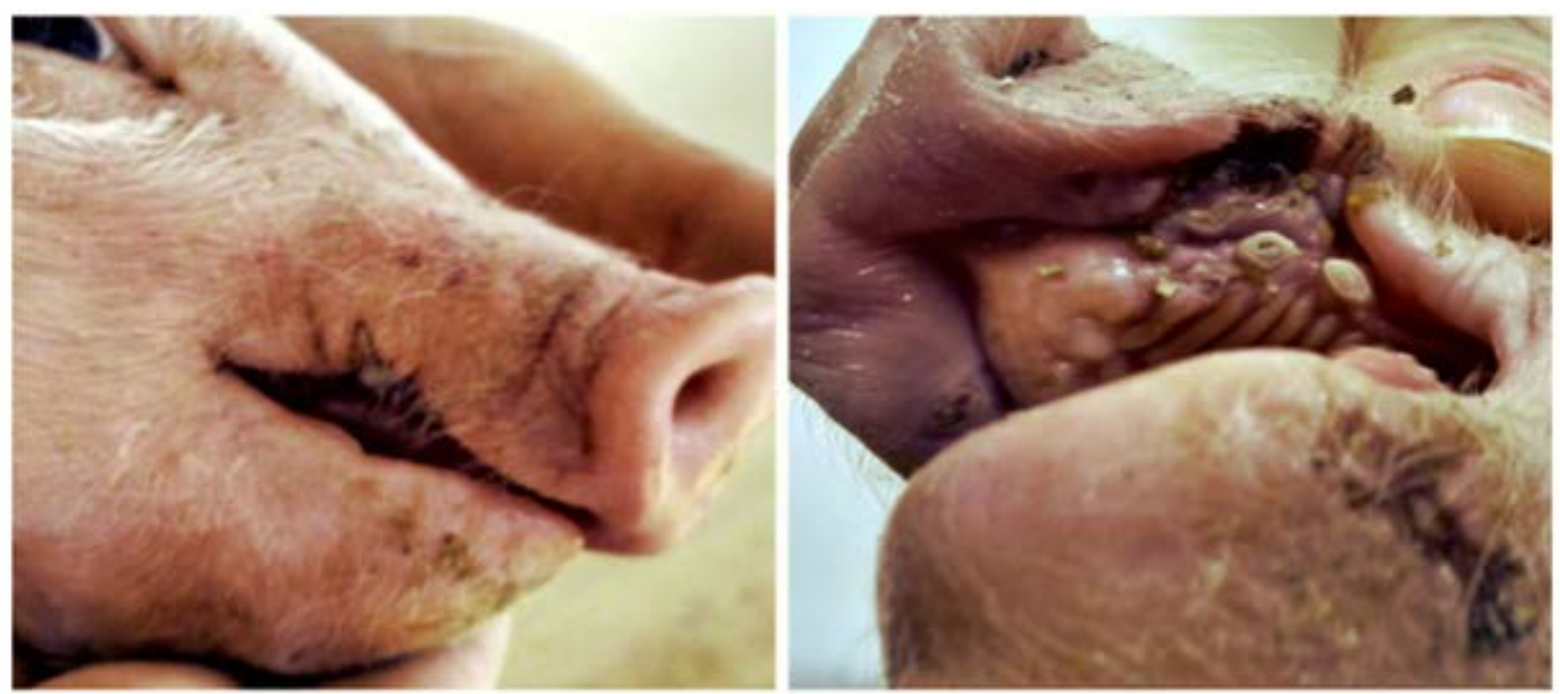

\section{Fonte: do autor}

Figura 1. Lesões encontradas nos lactentes no tratamento desgaste de dentes, decorrentes do manejo incorreto do desgaste dos dentes, na fase de maternidade.
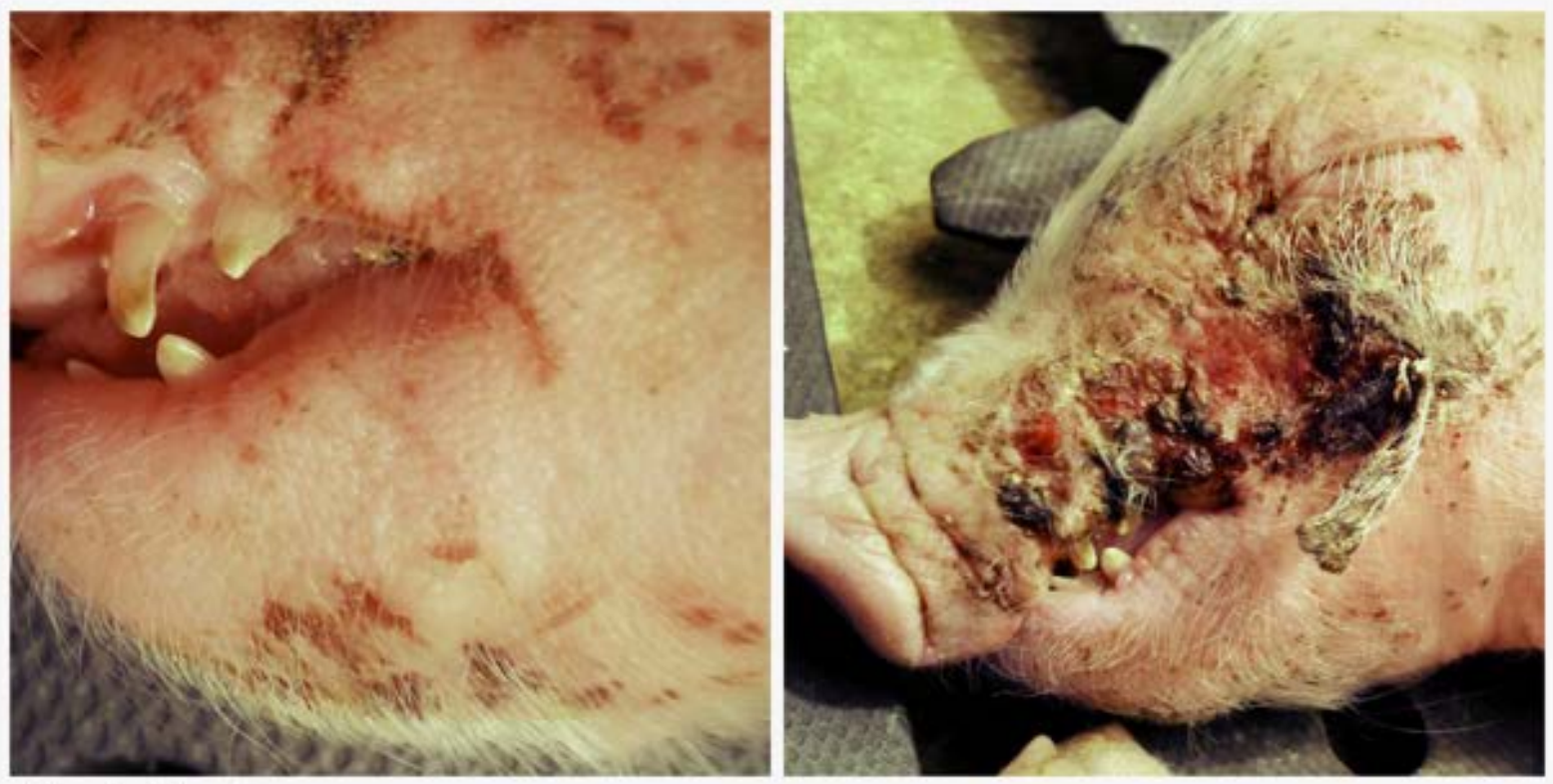

\section{Fonte: do autor}

Figura 2. Lesões encontradas nos lactentes sob o tratamento de manutenção dos dentes, decorrentes de disputas pelos tetos de maior produção de leite, na fase de maternidade.

Em relação aos tratamentos aplicados nos leitões, não foram encontrados efeitos significativos para o comportamento alimentar (comer e beber) das matrizes $(\mathrm{P}>0,05)$. Comer diferiu de beber, quando 
foi considerado o período avaliado $(\mathrm{P} \leq 0,05)$. Sobre os comportamentos agonísticos, estereotipados, exploratórios, lúdicos, dormir, deitar, sentada e em pé das matrizes, não foram encontradas diferenças significativas $(\mathrm{p}>0,05)$.

Normalmente, o comportamento agonístico das matrizes, contidas em gaiolas, era verificado no momento do manejo com os leitões, da alimentação, de limpeza e de retirada dos animais para o desmame, sobretudo por estarem presas e com movimentação limitada. A comum agressividade das matrizes decorre da interação com o homem. Porcas submetidas a manejo convencional em gaiolas costumam apresentar agressividade no momento da contensão dos leitões para manejo e no momento da retirada ao desmame. Segundo o Comitê Científico Veterinário da Comissão Europeia, matrizes suínas possuem preferência em manter contato social com outros suínos, demonstrando maior frequência de comportamentos lúdicos em relação à agressividade ${ }^{(33)}$. Devido a essas situações, consideradas como de rotina na granja, faz-se necessário debater um novo sistema racional das técnicas de manejo.

Neste estudo, o comportamento estereotipado da matriz foi observado a partir de atividades como morder os ferros das gaiolas, morder o piso de plástico e o cimento das gaiola e falsa mastigação. A função principal dessas estereotipias são compensar a frustração por não adaptação ao ambiente ${ }^{(28)}$. Neste estudo, não foram utilizados enriquecimentos ambientais nas instalações de maternidade, o que poderia reduzir a apresentação desse tipo de comportamento atípico da matriz. Dela Ricci et al. ${ }^{(34)}$, avaliando o comportamento de matrizes suínas na maternidade, em ambientes com e sem enriquecimentos, encontraram maiores frequências de comportamento estereotipado na ausência de enriquecimento ambiental. Confinados em baias ou presos por longo período, alguns animais apresentam comportamentos estereotipados, enquanto outros se tornam inativos e não responsivos ${ }^{(35)}$, demonstrando redução do bem-estar dos animais.

O comportamento de beber apresentado pela matriz teve uma segunda função, a distração, quando procuravam os bebedouros buscando se refrescar ou por atividade lúdica. Os tratamentos direcionados aos leitões, neste caso, não interferiram nos resultados ( $\mathrm{P}>0,05)$. O comportamento lúdico apresentou baixa frequência de apresentação, demonstrando que o bem-estar é pobre ${ }^{(36)}$ dentro da maternidade.

\section{Conclusões}

O procedimento de desgaste ou permanência dos dentes de leitões pode influenciar em comportamentos importantes, como o de mamar, responsável pelo eficiente ganho de peso. O desgaste dos dentes pode ser desnecessário diante dos ferimentos causados, em decorrência do manejo errôneo desta pratica.

São necessárias medidas preventivas como, por exemplo, a transferência de lactentes, no tempo ideal, entre fêmeas, para a permanência de leitões com dentes íntegros, buscando-se minimizar as disputas pelos tetos de maior quantidade de leite. 


\section{Referências}

1. Barcellos DESN. Etiological analysis of a problem of runt pigs in the nursing period, in an industrial pig herd. In: INTERNATIONAL PIG VETERINARY SOCIETY CONGRESS, 11., 1990, Lausanne, Switzerland. Proceedings... Lausanne, Switzerland: Swiss Association of Swine Medicine, 1990. p. 384.

2. Bates RO, Hoge MD, Edwards DB, Straw BE. The influence of canine teeth clipping on nursing and nursery pig performance. Journal of Swine Health and Production, 2003;11:75-79.

3. Sousa, P. Conforto térmico e bem-estar na suinocultura. I. Lavras: UFLA, 2004. 69 p.

4. Weary DM, Appleby MC, Fraser D. Responses of piglets to early separation from the sow. Applied Animal Behavioral Science, 1999; 63:289-300.

5. Pandorfi H, Silva IJO, Moura DJ, Sevegnan, KBN. Análise de imagem aplicada ao estudo do comportamento de leitões em abrigo escamoteador. Engenharia Agrícola, 2004;24:274-284.

6. EMBRAPA Suínos e Aves. Produção de Suínos. 2003. Disponível em: <http://sistemasdeproducao.cnptia.embrapa.br/FontesHTML/Suinos/SPSuinos/construcao.html>. Acesso: 01 maio 2015.

7. Kilgour R, Dalton S. Livestock Behaviour. London: Grana, 1984. 45 p.

8. Sobestiansky J, Wentz I, Silveira PRS, Sesti LCA. Suinocultura intensiva: produção, manejo e saúde do rebanho. Brasília: EMBRAPA, Sistema de Produção de Informação - SPI, 1998.

9. Lewis N J. Frustration of goal-directed behaviour in swine. Applied Animal Behaviour Science, v. 64, p. 19-29, 1999.

10. Grandin T, Johnson C. Bem-estar dos animais. São Paulo: Rocco, 2009. 336 p.

11. Sarubbi J. Bem estar animal não se restringe às instalações e equipamentos: o uso de novas tecnologias. In: FÓRUM INTEGRAL DE SUINOCULTURA: TEORIA E PRÁTICA DO BEM ESTAR ANIMAL NA PRODUÇÃO DE SUÍNOS, 1., 2011, Curitiba. Anais... 2011. p. 36-50.

12. Broom DM. Animal welfare: concepts and measurement. Journal of Animal Science, 1991;69:4167-4175.

13. CONSEJO DE LA UNIÓN EUROPEA. Directiva 2008/120/CE del Consejo de 18 de diciembre de 2008 relativa a las normas mínimas para la protección de cerdos (Versión codificada). Disponível em: http://eurlex.europa.eu/legal-content/ES/TXT/?uri=CELEX\%3A32008L0120. Acesso em 28/10/2015.

14. KOPPEN. Clima dos Municipios Paulistas. 2011. Disponível em: http://www.cpa.unicamp.br/outrasinformacoes/clima-dos-municipios-paulistas.html> Acesso: 03/08/2015.

15. Lewis E, Boyle L. The pros and cons of theeth clipping. 2003. Disponível em: <www.teagasc.ie/publications/2003/pigconf/paper02.html> Acesso em: 23 fev. 2015.

16. Koller FL. et al. Prevenção e tratamento da infecção urinária em matrizes suínas. Porto Alegre: UFRGS -

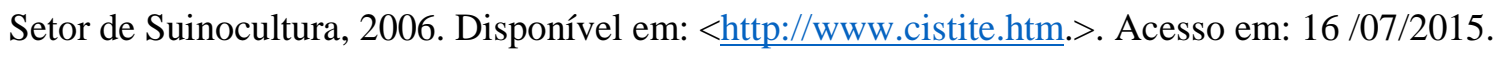

17. Bollwahn W. Klinische diagnostik der lahmheiten beim Schwein unter besonder berücksichtigung der Röntgenuntersuchung. 1965. 210 f. Tese (Livre docência) - Hannover: Tierärztliche Hochschule, 1965.

18. Schulze W. 1980. Klinische Untersuchungen, p.3-32. In: Schulze W, Bichhardt K, Bollwahn W, Michwitz GV \& Plonait H. (Eds), Klinik der Schweinekrankheiten. M. und H. Schaper, Hannover.

19. Straw BE, D’Allaire S, Mengeling WL, Taylor DJ. Diseases of Swine, 8th edition, Iowa State University 
Press, 1999.

20. Del Claro K. Comportamento animal: uma introdução à ecologia comportamental (Ed). Jundiaí. Livraria Conceito, 2004. 132 p.

21. Ferreira RA, Oliveira RFM, Donzele JL, Lopes DC, Dias UAD, Resende WO, Vieira Vaz RGM. Redução do nível de proteína bruta e suplementação de aminoácidos em rações para suínos machos castrados mantidos em ambiente termoneutro dos 30 aos 60 kg. Revista Brasileira de Zootecnia, 2005;34:548-556.

22. XAVIER LH. Modelos univariado e multivariado para análise de medidas repetidas e verificação da acurácia do modelo univariado por meio de simulação. 2000. 91 f. Dissertação (Mestrado em Agronomia) Escola Superior de Agricultura "Luiz de Queiroz", Piracicaba, 2000. Disponível em https://www.academia.edu/9814101/MODELOS UNIVARIADO E MULTIVARIADO PARA ANALISE DE MEDIDAS REPETIDAS E VERIFICAÇAO DA ACURACIA DO MODELO UNIVARIADO P OR_MEIO_DE_SIMULAÇ_O, Acesso em 24 maio de 2015.

23. Wu WZ, Wang XQ, Wu GY, Kim SW, Chen F, Wang JJ. Differential composition of proteomes in sow colostrum and milk from anterior and posterior mammary glans. Journal Animal Science, 2010;88:2657-2664.

24. Hartsock, TG, Graves HB. Neonatal behavior and nutrition-related mortality in domestic swine. Journal of Animal Science, 1976;42:235-241.

25. Jeppesen LE. Teat-order in groups of piglets reared on an artificial sow. I. Formation of teat-order and influence of milk yield on teat preference. Applied Animal Ethology, 1982;8:335-345.

26. Rosillon-Warnier A, Paquay R. Development and consequences of teat-order in piglets. Applied Animal Behaviour Science, 1984;13:47-58.

27. Sarubbi J. Bem-estar dos animais e uso racional de energia elétrica em sistemas de aquecimento para leitões desmamados. 2009. 190 p. Tese (Doutorado em Engenharia Agrícola) - Faculdade de Engenharia Agrícola, Universidade Estadual de Campinas, Campinas, 2009. Disponível em: http://www.bibliotecadigital.unicamp.br/document/?code=000443238, Acesso em 24 maio de 2015.

28. Dantzer R, Mormede P. De-arousal properties of stereotyped behaviour: evidence from pituitary-adrenal correlates in pigs. Applied Animal Behaviour Science, 1983;10:233-244.

29. Riesenberg A A. et al. Enriquecimento ambiental na forma de brinquedos para leitões após o desmame. Disponível

em: http://www.agraria.pro.br/sistema/index.php?journal=agraria\&page=article\&op=view\&path\%5B\%5D=agrar ia_v5i2a660\&path\%5B\%5D=710 Acesso em: 30 abr. 2013.

30. McBride G, James JW, Wyeth GSF. Social behaviour of domestic animals. VIII Variations in weaning weight in pigs. Animal Production, 1965:7:67-74.

31. Scheel DE, Graves HB, Sherritt GW. Nursing order, social dominance and growth in swine. Journal of Animal Science, 1977;45:219-229.

32. Ferreira, RA, Chiquieri J, Mendonça PP, Melo TV, Cordeiro MD, Soares RTRN. Comportamento e parâmetros fisiológicos de leitões nas primeiras 24 horas de vida, Ciência Agrotecnológica 2007;31:6.

33. Stevenson P. Questões de bem-estar animal na criação intensiva de suínos na união européia. In: CONFERENCIA INTERNACIONAL VIRTUAL SOBRE QUALIDADE DE CARNE,1., 2000, Concórdia. Anais... Concórdia, 2000. p. 4-5.

34. Dela Ricci G, Berto D, Sartori R, Dalla Costa OA, Lopes L. Análise do comportamento de matrizes suínas lactantes com o uso de enriquecimento ambiental na maternidade. JORNADA DE INICIAÇÃO CIENTÍFICA 
EMBRAPA, 6., E II SEMINÁRIO DE PESQUISA E EXTENSÃO DA UNC, 2., 2012. Anais... 2012.

35. Broom, DM. Biology of behaviour. Cambridge: Cambridge University Press, 1981. 35 p.

36. Broom DM, Molento CFM. Bem-estar animal: conceitos e questões relacionadas - Revisão. Archives of Veterinary Science, 2004;9:1-11. 